\title{
Research Article \\ The Use of Fractional Order Derivative to Predict the Groundwater Flow
}

\author{
Abdon Atangana ${ }^{1}$ and Necdet Bildik ${ }^{2}$ \\ ${ }^{1}$ Institute for Groundwater Studies, Faculty of Natural and Agricultural Sciences, University of the Free State, \\ P.O. Box 9300, Bloemfontein, South Africa \\ ${ }^{2}$ Department of Mathematics, Faculty of Art \& Sciences, Celal Bayar University, Muradiye Campus, \\ 45047 Manisa, Turkey
}

Correspondence should be addressed to Abdon Atangana; abdonatangana@yahoo.fr

Received 2 July 2013; Revised 27 August 2013; Accepted 3 September 2013

Academic Editor: Tirivanhu Chinyoka

Copyright (C) 2013 A. Atangana and N. Bildik. This is an open access article distributed under the Creative Commons Attribution License, which permits unrestricted use, distribution, and reproduction in any medium, provided the original work is properly cited.

\begin{abstract}
The aim of this work was to convert the Thiem and the Theis groundwater flow equation to the time-fractional groundwater flow model. We first derived the analytical solution of the Theim time-fractional groundwater flow equation in terms of the generalized Wright function. We presented some properties of the Laplace-Carson transform. We derived the analytical solution of the Theistime-fractional groundwater flow equation (TFGFE) via the Laplace-Carson transform method. We introduced the generalized exponential integral, as solution of the TFGFE. This solution is in perfect agreement with the data observed from the pumping test performed by the Institute for Groundwater Study on one of its borehole settled on the test site of the University of the Free State. The test consisted of the pumping of the borehole at the constant discharge rate $Q$ and monitoring the piezometric head for 350 minutes.
\end{abstract}

\section{Introduction}

Groundwater problem is perhaps one of the most difficult real-world problems to be modelled into mathematical formulation. To model this problem accurately, one must know precisely the behavior of the medium through which the water is moving. However, this medium through which the flow occurs can change from one point to another, also from one period to another. For example, the hydraulic conductivity of an aquifer can differ from one direction to another. Several scholars have intensively tried to propose a better model that can be used to predict the movement of water through the aquifer. However, their results still present some lacks. Recently, It was revealed that real problems modelled via fractional order derivative present better results when matching their mathematical representation with experimental data. To test this, Botha and Cloot [1] presented some good results by generalizing the groundwater flow equation to the concept of fractional order derivatives. In the same line of ideas, Atangana [2] examined an approximate solution of the generalized groundwater flow equation via the Frobenius method. The results obtained from his investigation showed better prediction. Recently, Atangana and Botha further extended the fractional groundwater equation to the concept of the fractional-variation order groundwater [3]. They presented the stability and the convergence of the numerical scheme via Crank-Nicolson method. Up to now, there is no approximate or exact analytical mathematical expression that can be used to describe the solution of the fractional groundwater flow equation. Therefore, one of the purposes of this work is to present some analytical mathematical expression than can be used as approximate solution of the time-fractional groundwater flow equation.

An aquifer test (or a pumping test) is conducted to evaluate an aquifer by "stimulating" the aquifer through constant pumping and observing the aquifer's "response" (drawdown) in observation wells. Aquifer testing is a common tool that hydrogeologists use to characterize a system of aquifers, aquitards, and flow system boundaries. Aquifer tests are typically interpreted by using an analytical model of aquifer flow (the most fundamental being the Theis solution) to 
match the data observed in the real-world then assuming that the parameters from the idealized model apply to the realworld aquifer. In more complex cases, a numerical model may be used to analyze the results of an aquifer test, but adding complexity does not ensure better results. For the most part frequently, an aquifer assessment is carried out by propelling water out from one borehole at a fixed speed and for as a minimum of 24 hours at the same time as cautiously evaluating the water levels in the observed borehole. When water is pumped from the pumping well, the pressure in the aquifer that feeds that well declines. This decline in pressure will show up as drawdown (change in hydraulic head) in an observation well. Drawdown decreases with radial distance from the pumping well and drawdown increases with the length of time that the pumping continues. The aquifer characteristics which are evaluated by most aquifer tests are $[4,5]$ as follows.

(i) The hydraulic conductivity is the rate of flow of water through a unit crosses sectional area of an aquifer at a unit hydraulic gradient. In English units the rate of flow is in gallons per day per square foot of cross sectional area.

(ii) Specific storage or storativity being a measure of the amount of water a confined aquifer will give up for a certain change in head.

(iii) The transmissivity is the rate at which water is transmitted through a unit thickness of an aquifer under a unit hydraulic gradient. It is equal to the hydraulic conductivity times the thickness of an aquifer.

The rest of this paper has been presented as follows. In Section 1, we presented the background of the fractional order derivative. We derived the analytical solution of the Theim fractional groundwater flow equation in Section 3. In Section 4, we presented the derivation of the Theis fractional groundwater flow via the Laplace-Carson transform method. We presented an alternative analytical solution of the Theis fractional groundwater flow equation in terms of the generalized exponential integral in Section 5. The numerical comparisons with experimental data are presented in Section 6 and the conclusion is in Section 7. We will start with the background of the fractional derivative.

\section{Background of the Fractional Order Derivative}

There exists the vast literature on different definitions of fractional derivatives. The most popular ones are the RiemannLiouville and the Caputo derivatives [6-11]. Caputo's definition has the form of

$$
\begin{aligned}
D_{x}^{\alpha}(f(x))= & \frac{1}{\Gamma(n-\alpha)} \frac{d^{n}}{d x^{n}} \\
& \times \int_{0}^{x}(x-t)^{n-\alpha-1} f(t) d t, \quad n-1 \leq \alpha \leq n .
\end{aligned}
$$

For the case of the Caputo fractional order derivative, we have the following definition:

$$
\begin{aligned}
D_{x}^{\alpha}(f(x))= & \frac{1}{\Gamma(n-\alpha)} \\
& \times \int_{0}^{x}(x-t)^{n-\alpha-1} \frac{d^{n}}{d x^{n}} f(t) d t, \quad n-1 \leq \alpha \leq n .
\end{aligned}
$$

Each of the pervious fractional order derivatives presents some advantages and disadvantages [6-10]. The RiemannLiouville derivative of a constant is not zero while Caputo derivative of a constant is zero but demands higher conditions of regularity for differentiability [6-10]: to compute the fractional derivative of a function in the Caputo sense, we must first calculate its derivative [11]. Caputo derivatives are defined only for differentiable functions while functions that have no first-order derivative might have fractional derivatives of all orders less than one in the RiemannLiouville sense [12,13]. Guy Jumarie has recently modified the Riemann-Liouville derivative (see [14])

$$
D_{x}^{\alpha}(f(x))=\frac{1}{\Gamma(n-\alpha)} \frac{d^{n}}{d x^{n}} \times \int_{0}^{x}(x-t)^{n-\alpha-1}\{f(t)-f(0)\} d t .
$$

The Caputo fractional derivative will be considered in this work due to the applicability of the Caputo derivative in realworld problems [15].

\section{Thiem' Groundwater Flow Equation}

The Theim fractional groundwater flow equation is an ordinary differential equation given in the following. The equation describes the change in level of water as function of distance during the pumping test [5]:

$$
D_{r r}^{\alpha} \Phi(r)+\frac{1}{r} \Phi(r)=0, \quad 1<\alpha \leq 2 .
$$

Subject to the initial condition, $Q=2 \pi T D_{r}\left(\Phi\left(r_{b}\right)\right)$.

We will make use of the Laplace transform to derive analytical solution of (4). Thus, multiplying on both sides of (4) by $r$ and secondly applying the Laplace transform, we obtain the following expression:

$$
\begin{gathered}
\frac{d[L(\Phi)(s)]}{d s}+\left(\frac{\alpha}{s}+\frac{1}{s^{\alpha}}\right)(L(\Phi)(s)) \\
=\sum_{m=2}^{l} d_{m}(m-1) s^{m-2-\alpha},
\end{gathered}
$$

where $d_{m}=D_{0^{+}}^{\alpha-m} \Phi\left(0^{+}\right)(m=2, \ldots, l)$. Now, one can derive the solution of the ordinary order differential equation with respect to the Laplace transform of $\Theta(s)=L(\Phi(r))$ :

$$
\begin{aligned}
\Theta(s)= & s^{-\alpha} \exp \left[-\frac{s^{1-\alpha}}{1-\alpha}\right] \\
& \times\left[a_{1}+\sum_{m=2}^{l} d_{m}(m-1) \int s^{m-2} \exp \left[-\frac{s^{1-\alpha}}{1-\alpha}\right] d s\right],
\end{aligned}
$$


with $a_{1}$ an arbitrary real constant that will be obtained via the initial condition. We next expand the exponential function in the integrand in a series, and using term-by-term integration, we arrive at the following expression:

$$
\Theta(s)=c \Theta_{1}(s)+\sum_{m=2}^{l} d_{m}(m-1) \Theta_{m}^{*}(s)
$$

with, of course,

$$
\begin{gathered}
\Theta_{1}(s)=s^{-\alpha} \exp \left[-\frac{s^{1-\alpha}}{1-\alpha}\right], \\
\Theta_{m}^{*}(s)=s^{-\alpha} \exp \left[\frac{s^{1-\alpha}}{\alpha-1}\right] \sum_{j=0}^{\infty}\left(\frac{1}{1-\alpha}\right)^{j} \frac{s^{(1-\alpha) j+m-1}}{[(1-\alpha) j+m-1] j !} .
\end{gathered}
$$

Now, applying the inverse Laplace transform on $\Theta_{1}(s)$ and using the fact that

$$
s^{-[\alpha+(\alpha-1) j]}=L\left[\frac{r^{\alpha+(\alpha-1) j-1}}{\Gamma(\alpha+(\alpha-1) j)}\right]
$$

we obtain

$$
\Phi_{1}(r)=r^{\alpha-1} o \Psi_{1}\left[(\alpha, \alpha-1) \mid \frac{x^{\alpha-1}}{\alpha-1}\right]
$$

with $o \Psi_{1}[\cdot]$ the generalized Wright function [16] for $p=$ 1 and $q=2$. We next expand the exponential function $\exp \left[-s^{1-\alpha} /(1-\alpha)\right]$ in power series; multiplying the resulting two series; in addition of this if we consider the number $b_{k}(\alpha, m)$ defined for $\alpha>0, m=2, \ldots l,(\alpha \neq(p+m-1) / p, p \notin$ $\mathbb{N})$ and $k \in \mathbb{N}_{0}$,

$$
b_{k}(\alpha, m)=\sum_{p, j=0, \ldots k, p+j=k}^{l} \frac{(-1)^{q}}{p ! j !(1-\alpha) q+m-1} .
$$

The previous family of number possesses satisfies the following recursive formula:

$$
\frac{b_{k}(\alpha, m)}{b_{k+1}(\alpha, m)}=\frac{\alpha-m}{\alpha-1}+k
$$

which produces the explicit expression for $b_{k}(\alpha, m)$ in the form of

$$
b_{k}(\alpha, m)=\frac{\Gamma[(\alpha-m) /(\alpha-1)]}{(m-1) \Gamma[((\alpha-m) /(\alpha-1))+k]}, \quad k \in \mathbb{N}_{0} .
$$

Now, having the previous expression on hand, we can derive that

$$
\begin{aligned}
\Theta_{m}^{*}(s)= & s^{m-\alpha-1}\left(\sum_{j=0}^{\infty}\left(\frac{1}{1-\alpha}\right)^{j} \frac{s^{(1-\alpha) p}}{p !}\right) \\
& \times\left(\sum_{p=0}^{\infty}\left(\frac{1}{1-\alpha}\right)^{p} \frac{(-1)^{p}}{[(1-\alpha) p+m-1]} \frac{s^{(1-\alpha) j}}{p !}\right) \\
= & \sum_{k=0}^{\infty} b_{k}(\alpha, m)\left(\frac{1}{1-\alpha}\right)^{k} s^{(1-\alpha) k+m-\alpha-1} \quad(m=2, \ldots, l) .
\end{aligned}
$$

However, remembering (9) with $\beta=(\alpha-1) k+\alpha+1-m$, we can further derive the following expression for $\Phi_{m}^{*}(r)$ as

$$
\begin{aligned}
\Phi_{m}^{*}(r)= & \sum_{k=0}^{\infty} b_{k}(\alpha, m)\left(\frac{1}{1-\alpha}\right)^{k} \\
& \times \frac{\Gamma(k+1)}{\Gamma[\alpha+1-m+(\alpha-1) k]} \frac{x^{(\alpha-1) k+\alpha-m}}{k !}
\end{aligned}
$$

or in the simplified version we have

$$
\Phi_{m}^{*}(r)=\frac{\Gamma[(\alpha-m) /(\alpha-1)]}{(m-1)} \Phi_{m}(r)
$$

where

$$
\begin{aligned}
\Phi_{m}(r)= & r_{1}^{\alpha-m} \Psi_{2} \\
& \times\left[\begin{array}{c}
(1,1) \\
(\alpha+1-m, \alpha-1),\left(\frac{\alpha-m}{\alpha-1}, 1\right)
\end{array} \mid \frac{r^{\alpha-1}}{\alpha-1}\right] .
\end{aligned}
$$

It follows that the solution of the fractional-Thiem groundwater flow equation is in the form of

$$
\begin{aligned}
\Phi(r)= & a_{1} r^{\alpha-1} o \Psi_{1}\left[(\alpha, \alpha-1) \mid \frac{x^{\alpha-1}}{\alpha-1}\right] \\
& +a_{2} \sum_{m=2}^{l} b_{m}(m-1) \frac{\Gamma[(\alpha-m) /(\alpha-1)]}{(m-1)} r^{\alpha-m} \\
& \times{ }_{1} \Psi_{2}\left[(\alpha+1-m, \alpha-1),\left(\frac{\alpha-m}{\alpha-1}, 1\right) \mid \frac{r^{\alpha-1}}{\alpha-1}\right] .
\end{aligned}
$$

In our case $l=2$.

\section{Time-Fractional Theis Groundwater Flow Equation}

The easiest sweeping statement of subsurface water flow equation, which while we are on the subject is in addition in harmony in the midst of the real physics of the observed fact, is to presume that water level is not in a balanced state but momentary state. Theis (1935) [17] was the first to develop a formula for unsteady-state flow that introduces the time factor and the storativity. He noted that when a well-penetrating extensive confined aquifer is pumped at a constant rate, the influence of the discharge extends outward with time. The rate of decline of head, multiplied by the storativity and summed over the area of influence, equals the discharge. The unsteady-state (or Theis) equation, which was derived from the analogy between the flow of groundwater and the conduction of heat, is perhaps the most widely used partial differential equation in groundwater investigations:

$$
S D_{t} \Phi(r, t)=T D_{r r} \Phi(r, t)+\frac{1}{r} D_{r} \Phi(r, t)
$$


The aforementioned equation is classified under parabolic equation. To include explicitly the variability of the medium through which the flow takes place, the standard version of the partial derivative respect to time is replaced here with time-fractional order derivative to obtain

$$
S D_{t}^{\alpha} \Phi(r, t)=T D_{r r} \Phi(r, t)+\frac{1}{r} D_{r} \Phi(r, t), \quad 0<\alpha \leq 1
$$

with initial condition $\Phi(r, 0)=0$ and boundary condition $\lim _{r \rightarrow \infty} \Phi(r, t)=0, Q=2 \pi T \partial r \Phi\left(r_{b}, t\right)$, here $T$ is the transmissivity of the aquifer, $r_{b}$ is the ratio of the borehole, and $Q$ is the discharge rate, or the rate at which the water is being taken out of the aquifer.

Some few integral transform operators have been intensively used to solve some kind of ordinary and partial differential equations. See, for instance, the Fourier transform, the Laplace transform, the Mellin transform [18], and the Sumudu transform [19-22]. Beside these integral operators, there exists a similar operators called the Laplace-Carson transform [23]; this operator has been neglected. However, this operator has some properties that can be used to solve a kind of ODE, PDE, FODE, and FPDE. The aim of this section is therefore devoted to the discussion underpinning the definition, properties of the Laplace-Carson transform, and its application to the fractional groundwater flow equation. We shall start with the definition and properties.

Definition 1. Let $f(x)$ be a continuous function over an open interval $(0, \infty)$ such that its Laplace transform is $n$ time differentiable; then the Laplace-Carson transform $f$ is defined as follows:

$$
L_{c}(s)=L_{c}[f(x)](s)=\int_{0}^{\infty} x e^{-x s} f(x) d x
$$

and the inverse Laplace-Carson transform is defined as

$$
\begin{aligned}
f(x) & =L_{c}^{-1}\left[L_{c}[f(x)]\right] \\
& =\frac{-1}{2 \pi i} \int_{\alpha-i \infty}^{\alpha+i \infty} e^{s x}\left[-1\left[\int_{0}^{s} L_{c}(t)+F(0)\right]\right] d s,
\end{aligned}
$$

where $F(s)$ is the Laplace transform of $f(x)$. Before we continue, we shall prove that the previous definition is indeed the inverse inverse Laplace-Carson. In fact, from the definition of inverse Laplace-Carson of a function $f(x)$, we have that

$$
L_{c}(s)=L_{c}[f(x)](s)=\int_{0}^{\infty} x e^{-x s} f(x) d x=-\frac{d F(s)}{d s}
$$

thus,

$$
\int_{0}^{s} L_{c}(t) d t=-[F(s)-F(0)]
$$

It follows that

$$
\begin{aligned}
& \frac{-1}{2 \pi i} \int_{\alpha-i \infty}^{\alpha+i \infty} e^{s x}\left[-1\left[\int_{0}^{s} L_{c}(t) d t+F(0)\right]\right] d s \\
& =\frac{-1}{2 \pi i} \int_{\alpha-i \infty}^{\alpha+i \infty} e^{s x}[-[F(s)]] d s \\
& L_{c}^{-1}\left[L_{c}[f(x)]\right]=\frac{(-1)^{2}}{2 \pi i} \int_{\alpha-i \infty}^{\alpha+i \infty} e^{s x}[[F(s)]] d s=f(x) .
\end{aligned}
$$

Therefore, the inverse inverse Laplace-Carson is well defined.

\section{Some Properties of Laplace-Carson Transform}

In this part of the section, we consider some of the properties of the inverse Laplace-Carson that will enable us to find further transform pairs $\left\{f(x), L_{c}(s)\right\}$ without having to compute and consider the following:

$$
\begin{aligned}
& \text { (I) } L_{c}[s+c]=M_{n}\left[e^{-c x} f(x)\right] \\
& \text { (II) } L_{c}[f(a x)](s)=\frac{1}{a} L_{c}\left[\frac{s}{a}\right] \\
& \text { (III) } \int_{\alpha-i \infty}^{\alpha+i \infty} e^{s x} L_{c}(s) d s=x f(x)
\end{aligned}
$$

(IV) $L_{c}[a f(x)+b g(x)](s)$

$$
=\left[a L_{c}(f(x))+b L_{c}(g(x))\right](s)
$$

$$
\text { (V) } L_{c}\left[\frac{f(x)}{x}\right](s)=L[f(x)](s)
$$

(VI) $L_{c}[f(x) * h(x)](s)$

$$
=-\left[\frac{d F(s)}{d s} G(s)+\frac{d G(s)}{d s} F(s)\right]
$$

$$
\text { (VII) } \begin{aligned}
& L_{c}\left[\frac{d^{n} f(x)}{d x^{n}}\right](s) \\
&=-\left[n s^{n-1} F(s)+s^{n} \frac{d F(s)}{d s}\right. \\
&\left.-\sum_{k=0}^{n-2}(n-k-1) s^{n-k-2} \frac{d^{k} f(0)}{d x^{k}}\right] .
\end{aligned}
$$

Let us verify the previous properties. We shall start with (I), by definition, we have the following:

$$
\begin{aligned}
L_{c}\left[e^{-c x} f(x)\right] & =\int_{0}^{\infty}\left[x e^{-c x} e^{-s x} f(x)\right] d x \\
& =\int_{0}^{\infty}\left[x e^{-(c+s) x} f(x)\right] d x=L_{c}[s+c]
\end{aligned}
$$

and then the first property is verified. 
For (II) we have the following by definition:

$$
\begin{aligned}
& L_{c}[f(a x)](s) \\
& \quad=\int_{0}^{\infty}\left[x e^{-x s} f(a x)\right] d x=-\frac{d}{d s}[L[f(a x)](s)] .
\end{aligned}
$$

Now, using the property of the Laplace transform $L[f(a x)](s)=(1 / a) F(s / a)$, we can further obtain

$$
\begin{aligned}
L_{c}[f(a x)](s) & =-\frac{d}{d s}\left[\frac{1}{a} F\left(\frac{s}{a}\right)\right] \\
& =\frac{1}{a}(-1) \frac{d}{d s}\left[F\left(\frac{s}{a}\right)\right]=\frac{1}{a} L_{c}\left[\frac{s}{a}\right]
\end{aligned}
$$

and then, the property number (II) is verified.

For number (III), we have the following: Let $g(x)=$ $x f(x)$; then

$$
\begin{aligned}
\int_{\alpha-i \infty}^{\alpha+i \infty} e^{s x} L_{c}(s) d s & =\int_{\alpha-i \infty}^{\alpha+i \infty} e^{s x}\left[\int_{0}^{\infty} e^{-x s} x f(x) d x\right] d s \\
& =\int_{\alpha-i \infty}^{\alpha+i \infty} e^{s x}\left[\int_{0}^{\infty} e^{-x s} g(x) d x\right] d s .
\end{aligned}
$$

By the theorem of inverse Laplace transform, we obtain

$$
\int_{\alpha-i \infty}^{\alpha+i \infty} e^{s x} M_{n}(s) d s=g(x)=x f(x) .
$$

Number (IV) and (V) are obvious to be verified. For number (VI), we have the following by definition:

$$
\begin{aligned}
L_{c}[f(x) * h(x)](s) \\
\quad=\int_{0}^{\infty}\left[x e^{-s x} f(x) * h(x)\right] \\
=-\frac{d}{d s}[L(f(x) * h(x))(s)] .
\end{aligned}
$$

Now, using the property of Laplace transform of the convolution, we obtain the following:

$$
L(f(x) * h(x))(s)=F(s) \cdot G(s)
$$

and then, using the property of the derivative for the product of two functions, we obtain

$$
\begin{aligned}
L_{c}[f(x) * h(x)](s) & =-\frac{d}{d s}[F(s) \cdot G(s)] \\
& =-\left[\frac{d F(s)}{d s} G(s)+\frac{d G(s)}{d s} F(s)\right] .
\end{aligned}
$$

And then, the property number (VI) is verified.

For number (VII), by definition, we have the following:

$$
\begin{aligned}
L_{c}\left[\frac{d^{n} f(x)}{d x^{n}}\right](s) & =\int_{0}^{\infty}\left[x e^{-s x} \frac{d^{n} f(x)}{d x^{n}}\right] d x \\
& =-\frac{d}{d s}\left[L\left(\frac{d^{n} f(x)}{d x^{n}}\right)(s)\right] .
\end{aligned}
$$

Now, using the property of the Laplace transform,

$$
L\left(\frac{d^{n} f(x)}{d x^{n}}\right)(s)=s^{n} F(s)-\sum_{k=0}^{n-1} s^{n-k-1} \frac{d^{k} f(0)}{d x^{k}} .
$$

Now, deriving the previous expression $n$-time, we obtain the following expression:

$$
\begin{aligned}
-\frac{d}{d s}[ & \left.s^{n} F(s)-\sum_{k=0}^{n-1} s^{n-k-1} \frac{d^{k} f(0)}{d x^{k}}\right] \\
= & -\left[n s^{n-1} F(s)+s^{n} \frac{d F(s)}{d s}\right. \\
& \left.-\sum_{k=0}^{n-2}(n-k-1) s^{n-k-2} \frac{d^{k} f(0)}{d x^{k}}\right] .
\end{aligned}
$$

This completes the proof of number (VI). We shall now use some properties of the Laplace-Carson transform to solve the fractional groundwater flow equation. To achieve this, we shall start by assuming that the solution of the main equation can be separated as follows:

$$
\Phi(r, t)=\Phi_{1}(t) \Phi_{2}(r) ;
$$

then, the separated equations become

$$
\begin{gathered}
{ }_{o}^{C} D_{t}^{\alpha} \Phi_{1}(t)+\lambda^{2} \Phi_{1}(t)=0, \\
D_{r r} \Phi_{2}(r)+\frac{1}{r} D_{r} \Phi_{2}(r)+\lambda^{2} \Phi_{2}(r)=0,
\end{gathered}
$$

where $\lambda$ is the separation constant.

The first equation (45) can be solve directly by applying on both sides the Laplace transform to obtain

$$
L\left(\Phi_{1}(t)\right)=\Theta_{1}(s)=\frac{s^{\alpha-1}}{s^{\alpha}+\lambda^{2}} .
$$

Using the inverse formula of Laplace transform of twoparameter Mittag-Leffler function, we get

$$
\Phi_{1}(t)=c E_{\alpha, 1}\left(-\frac{S}{T} \lambda^{2} t^{\alpha}\right)
$$

where the Mittag-Leffler function is defined as follows:

$$
E_{\alpha, 1}(t)=\sum_{n=0}^{\infty} \frac{t^{\alpha n}}{\Gamma[\alpha n+1]} .
$$

To solve the second equation, we make use of the LaplaceCarson presented earlier, to obtain

$$
\begin{aligned}
-D_{s} & {\left[s^{2} \Theta_{2}(s)-s \Phi_{2}(0)-\frac{d \Phi_{2}(0)}{d r}\right] } \\
& +s \Theta_{2}(s)-\Phi_{2}(0)-D_{s} \Theta_{2}(s)=0 .
\end{aligned}
$$

Deriving, we obtain the following ordinary differential equation:

$$
D_{s} \Theta_{2}(s)\left[s^{2}+\lambda^{2}\right]=-s \Theta_{2}(s)
$$


for which the exact solution is given as

$$
\Theta_{2}(s)=\frac{1}{\sqrt{s^{2}+\lambda^{2}}}
$$

Now applying the inverse Laplace transform operator on both sides in the previous equation, we obtain the following in terms of the Bessel function first kind [24]:

$$
\Phi_{1}(r)=J_{0}(\lambda r)
$$

where the Bessel function first kind is defined as

$$
J_{0}(r)=\sum_{k=0}^{\infty} \frac{(-1)^{k}}{k !} \frac{1}{\Gamma(k+1)}\left(\frac{r}{2}\right)^{2 k} .
$$

Therefore, the solution of the fractional groundwater flow equation is given as

$$
\Phi(r, t)=c \sum_{n=0}^{\infty} E_{\alpha, 1}\left(-\frac{S}{T} \lambda_{n}^{2} t^{\alpha}\right) J_{0}\left(\lambda_{n} r\right) .
$$

Making use of the initial and boundary conditions, we obtain the constant $c$ to be

$$
c=\frac{Q}{4 \pi T}
$$

Then,

$$
\Phi(r, t)=\frac{Q}{4 \pi T} \sum_{n=0}^{\infty} E_{\alpha, 1}\left(-\frac{S}{T} \lambda_{n}^{2} t^{\alpha}\right) J_{0}\left(\lambda_{n} r\right) .
$$

We shall present an alternative solution in the next section via the Boltzmann variable method. By using the boundary condition, we can determine the Eigen value of (55).

\section{An Alternative Derivation of the Time-Fractional Groundwater Equation}

In this section, we present an alternative approximate solution of the groundwater flow equation. A method frequently used to derive some kind of parabolic partial differential equations is the so-called Boltzmann transformation [5], defined for an arbitrary $t_{0}<t$ by equation.

$$
u_{0}=\frac{S r^{2}}{4 T\left(t-t_{0}\right)} \text {. }
$$

Let us consider now the following function:

$$
\Phi(r, t)=\frac{c}{\left(t-t_{0}\right)} E_{\alpha, 1}\left[-u_{0}\right]
$$

with $c$ an arbitrary constant. If we assume that $r_{b}$ is the ratio of the borehole from which the water is taken out of the aquifer, then the total volume of the water withdrawn from the aquifer is given by

$$
Q_{0} \Delta t_{0}=4 \pi c T
$$

Hence,

$$
\Phi(r, t)=\frac{Q_{0} \Delta t_{0}}{4 \pi T\left(t-t_{0}\right)} E_{\alpha, 1}\left[-u_{0}\right]
$$

is the drawdown that will be observed at a distance, $r$, from the pumped borehole after the period $\Delta t_{0}$.

Now suppose that the a previous procedure is repeated $n$ times; that is, water is withdrawn for a short period of time, $\Delta t_{k}$, at a consecutive times, $t_{k+1}=t_{k}+\Delta t_{k},(k=0,1, \ldots, n)$. Now, since the fractional groundwater flow equation is linear differential equation, the total drawdown at any time $t>t_{n}$ will be given by

$$
\Phi(r, t)=\frac{1}{4 \pi T} \sum_{k=0}^{n} \frac{Q_{k} \Delta t_{k}}{4 \pi T\left(t-t_{k}\right)} E_{\alpha, 1}\left[-u_{k}\right] .
$$

Therefore, if $\Delta t \rightarrow 0$, the definition of the defined integral can be invoked to write

$$
\Phi(r, t)=\frac{1}{4 \pi T} \int_{t_{0}}^{t} \frac{Q(\tau) d \tau}{(t-\tau)} E_{\alpha, 1}\left[-\frac{S r^{2}}{4 T(t-\tau)}\right] d \tau
$$

However, using the Boltzman variable, we arrive at the following expression:

$$
\begin{gathered}
y=\frac{S r^{2}}{4 T(t-\tau)}, \\
\Phi(r, t)=\frac{1}{4 \pi T} \int_{t_{0}}^{\infty} \frac{Q(y)}{y} E_{\alpha, 1}[-y] d y .
\end{gathered}
$$

The previous solution will be called the most generalized general solution of fractional groundwater equation. However, this solution can be simplified somewhat under certain conditions. A particularly important solution which arises when $t_{0}$ is taken at zero and $Q(t)$ is a constant independent of time, and then we arrive at

$$
\Phi(r, t)=\frac{Q}{4 \pi T} \int_{u}^{\infty} \frac{1}{y} E_{\alpha, 1}[-y] d y=\frac{Q}{4 \pi T} W_{\alpha}(u)
$$

Here

$$
W_{\alpha}(u)=\int_{u}^{\infty} \frac{1}{y} E_{\alpha, 1}[-y] d y
$$

will be called the generalized exponential integral. It is worth pointing out that if alpha is equal to 1 , we recover the exact analytical solution of the groundwater flow equation proposed by Theis. Alternative iterations method $[8,11$, 20] can be used to derive approximate solutions of these problems.

\section{Numerical Simulations}

In this section, we investigate the behavior of the analytical solutions of the Theim fractional and the Theis fractional groundwater flow equation. We compare the analytical 


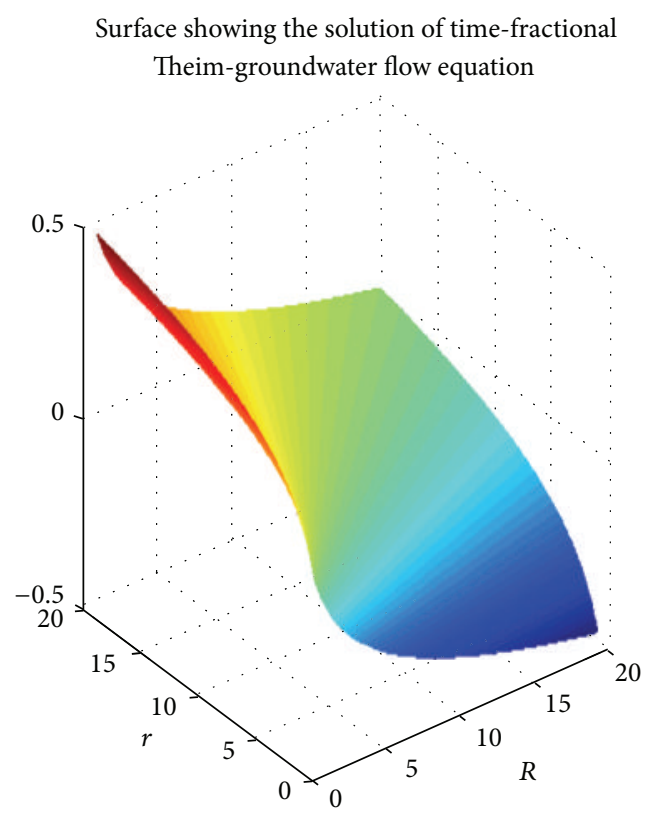

(a)

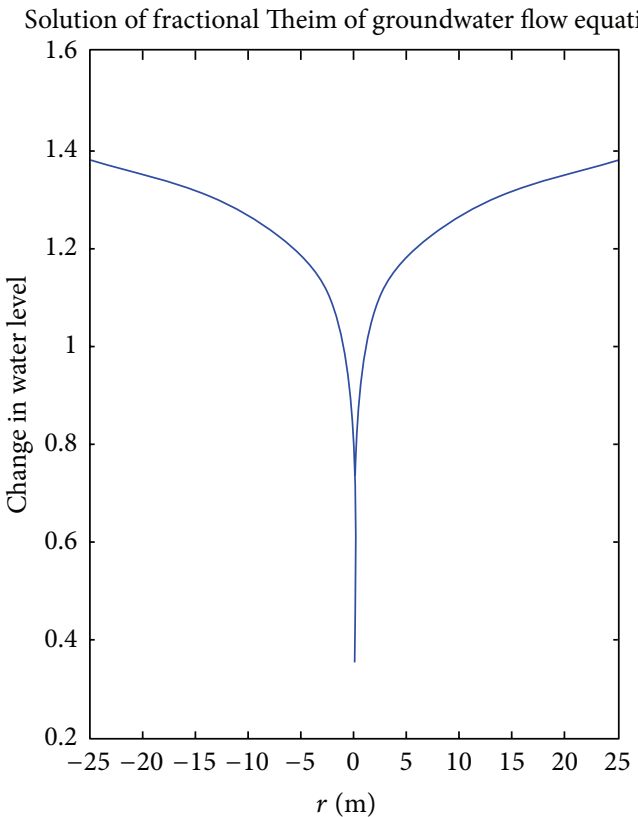

(c)
Solution of fractional Theim of groundwater flow equation

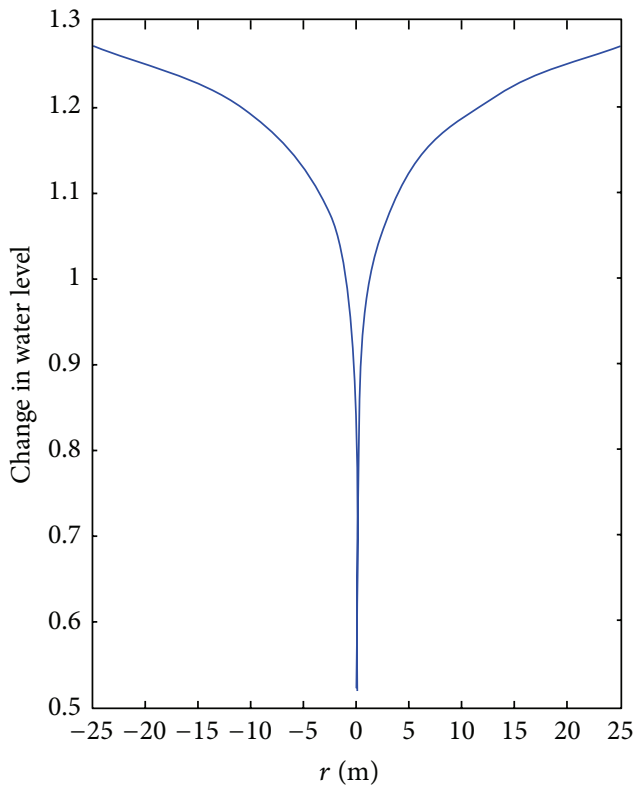

(b)

Solution of fractional Theim of groundwater flow equation

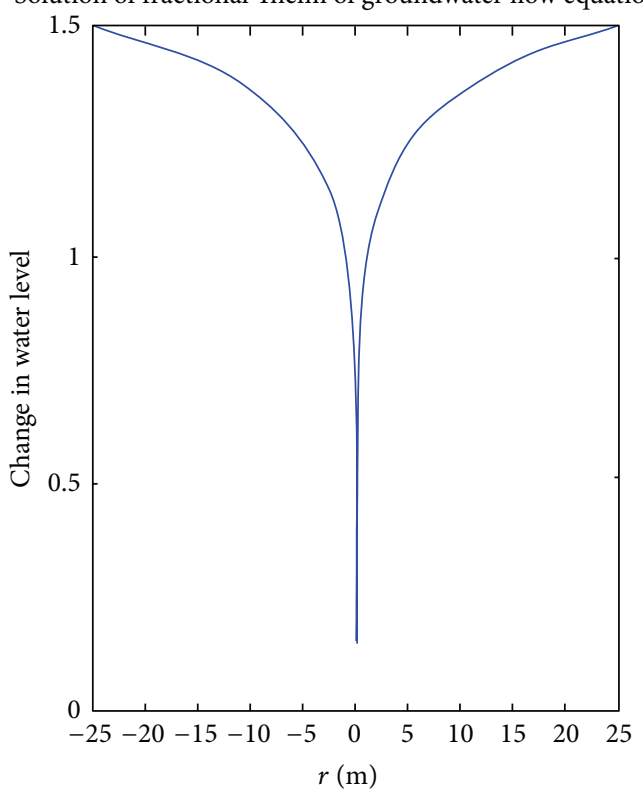

(d)

Figure 1: Numerical simulation of the Theim-time-fractional order groundwater flow equation, ((a) showing the surface for $\alpha=0.95)$; ((b) $\alpha=0.75)$; ((c) $\alpha=0.65)$ and ((d) $\alpha=0.55)$.

solution with the experimental data from the pumping test obtained from the experimental site of the Institute for Groundwater Studies, the University of the Free State, Bloemfontein Campus, South Africa. We shall start with the simulation of Theim fractional groundwater flow equation. The analytical solution of the main problem was depicted in Figures 1(a), 1(b), 1(c), and 1(d). It is worth to mention from the figures that the order of the derivative plays an important role in the simulation.
We shall present the numerical solution for the Theistime-fractional groundwater flow equation for a fixed distance. The analytical solution of the main problem was depicted in Figures 2(a), 2(b), 2(c), and 2(d).

We shall present in Figure 3 the comparison of the analytical solution of the Theis-time-fractional ground water flow equation, with the experimental data from the pumping test performed by the Institute for Groundwater Study on one of its borehole settled on the test site of the University of the 


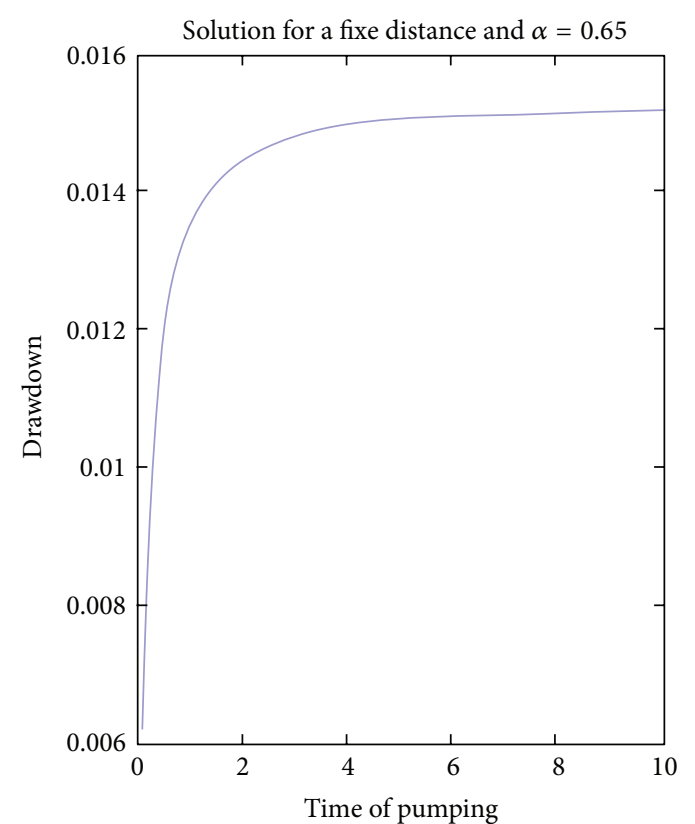

(a)

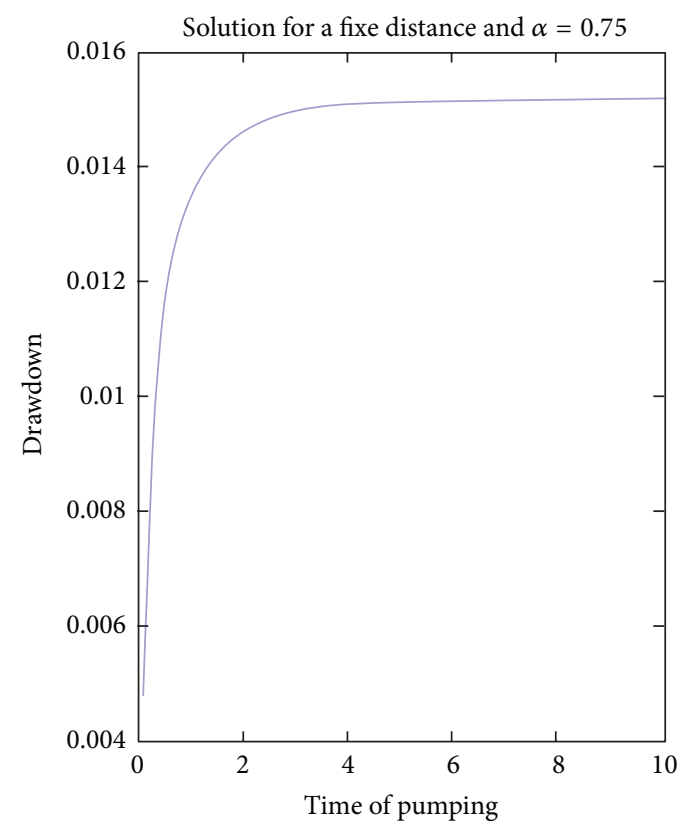

(c)

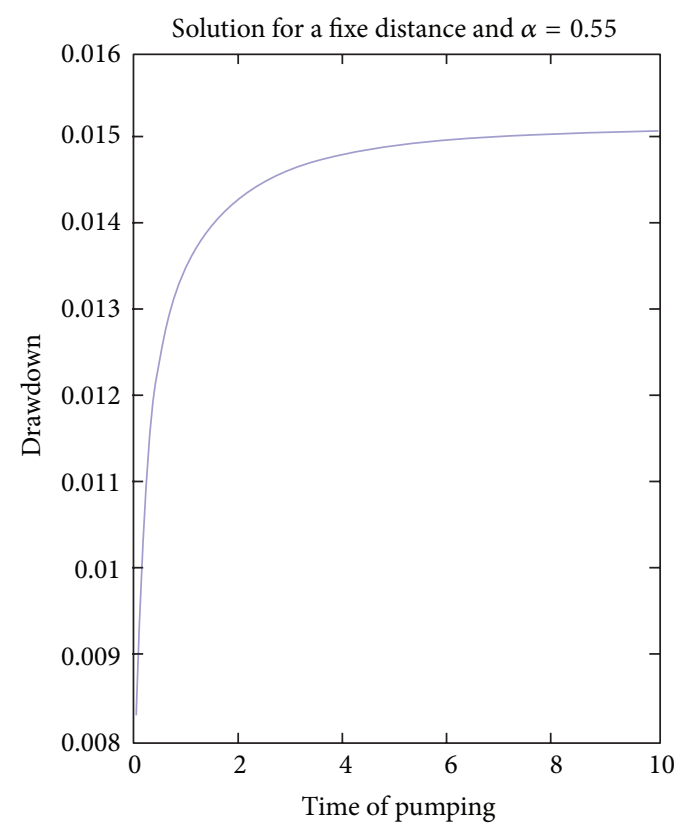

(b)

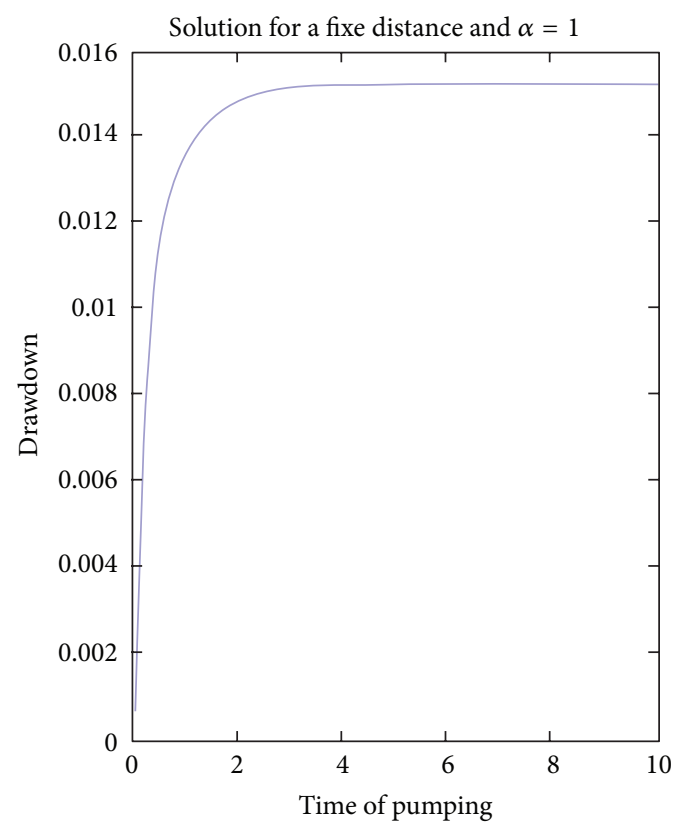

(d)

FIGURE 2: Numerical representation of (66) for different values of alpha.

Free State. The test consisted of the pumping of the borehole at the constant discharge rate $Q=4.5$ and monitoring the piezometric head for 350 minutes, at the distance of $r=$ $31.54 \mathrm{~m}$.

A Chinese proverb says, I quote "An image is equivalent to ten thousand words." With any reservation we can conclude that the fractional order derivative plays an important role while modeling real-world problem into mathematical equation. This solution is in perfect agreement with the data observed from the pumping test performed by the institute for groundwater study on one of their borehole settled on the test site of the University of the Free State.

\section{Conclusion}

We have generalized to Theim and Theis groundwater flow equation to the concept of fractional order derivatives. The resulting equations were solved analytically via several techniques, including the Laplace transform method, the Fourier transform method, the Laplace-Carson transform 


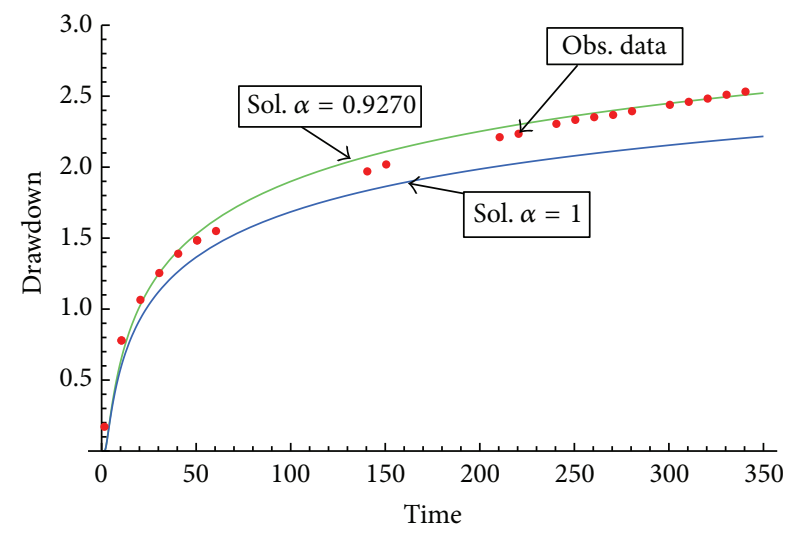

FIgURE 3: Comparison of Theis-time-fractional groundwater flow equation with experimental data from real observation.

method, and the Boltzmann variable method. The numerical simulations show that the fractional order derivative plays an important role in the simulation process. In addition, we compare the analytical solution with experimental data to access the accuracy of the fractional groundwater model. The analytical solution was in perfect agreement with experimental data.

\section{References}

[1] J. F. Botha and A. H. Cloot, "A generalised groundwater flow equation using the concept of non-integer order derivatives," Water SA, vol. 32, no. 1, pp. 1-7, 2006.

[2] A. Atangana, "Numerical solution of space-time fractional order derivative of groundwater flow equation," in Proceedings of the International Conference of Algebra and Applied Analysis, p. 20, Istanbul, Turkey, June 2012.

[3] A. Atangana and J. F. Botha, "Generalized groundwater flow equation using the concept of variable order derivative," Boundary Value Problems, vol. 2013, article 53, 2013.

[4] J. Boonstra and R. A. L. Kselik, SATEM 2002: Software for Aquifer Test Evaluation, International Institute for Land Reclamation and Improvement, Wageningen, The Netherlands, 2002.

[5] G. P. Kruseman and N. A. de Ridder, Analysis and Evaluation of Pumping Test Data, International Institute for Land Reclamation and Improvement, Wageningen, The Netherlands, 2nd edition, 1990.

[6] H. Jafari and C. M. Khalique, "Homotopy perturbation and variational iteration methods for solving fuzzy differential equations," Communications in Fractional Calculus, vol. 3, no. 1, pp. 38-48, 2012.

[7] S. Duan, R. Rach, D. Buleanu, and A. M. Wazwaz, "A review of the Adomian decomposition method and its applications to fractional differential equations," Communications in Fractional Calculus, vol. 3, no. 2, pp. 73-99, 2012.

[8] A. Atangana and E. Alabaraoye, "Solving system of fractional partial differential equations arisen in the model of HIV infection of CD4+ cells and attractor one-dimensional KellerSegel equation," Advances in Difference Equations, vol. 2013, article 94, 2013.
[9] D. Baleanu, K. Diethelm, E. Scalas, and J. J. Trujillo, Fractional Calculus Models and Numerical Methods, Series on Complexity, Nonlinearity and Chaos, World Scientific, Singapore, 2012.

[10] A. Atangana and A. Secer, "A note on fractional order derivatives and table of fractional derivatives of some special functions," Abstract and Applied Analysis, vol. 2013, Article ID 279681, 8 pages, 2013.

[11] G. C. Wu, "New trends in the variational iteration method," Communications in Fractional Calculus, vol. 2, pp. 59-75, 2011.

[12] G. Jumarie, "On the representation of fractional Brownian motion as an integral with respect to (dt)a," Applied Mathematics Letters, vol. 18, no. 7, pp. 739-748, 2005.

[13] G. Jumarie, "Modified Riemann-Liouville derivative and fractional Taylor series of nondifferentiable functions further results," Computers and Mathematics with Applications, vol. 51, no. 9-10, pp. 1367-1376, 2006.

[14] G. Jumarie, "Table of some basic fractional calculus formulae derived from a modified Riemann-Liouville derivative for nondifferentiable functions," Applied Mathematics Letters, vol. 22, no. 3, pp. 378-385, 2009.

[15] A. Atangana, "New class of boundary value problems," Information Sciences Letters, vol. 1, no. 2, pp. 67-76, 2012.

[16] S. G. Samko, A. A. Kilbas, and O. I. Marichev, Fractional Integrals and Derivatives, Translated from the 1987 Russian Original, Gordon and Breach, Yverdon, Switzerland, 1993.

[17] C. V. Theis, "The relation between the lowering of the piezometric surface and the rate and duration of discharge of a well using ground-water storage," Transactions, American Geophysical Union, vol. 16, no. 2, pp. 519-524, 1935.

[18] P. Flajolet, X. Gourdon, and P. Dumas, "Meilin transforms and asymptotics: harmonic sums," Theoretical Computer Science, vol. 144, no. 1-2, pp. 3-58, 1995.

[19] G. K. Watugala, "Sumudu transform: a new integral transform to solve differential equations and control engineering problems," International Journal of Mathematical Education in Science and Technology, vol. 24, pp. 35-43, 1993.

[20] A. Atangana and A. Kılıçman, "The use of Sumudu transform for solving certain nonlinear fractional heat-like equations," Abstract and Applied Analysis, vol. 2013, Article ID 737481, 12 pages, 2013.

[21] M. G. M. Hussain and F. B. M. Belgacem, "Transient solutions of Maxwell's equations based on sumudu transform," Progress in Electromagnetics Research, vol. 74, pp. 273-289, 2007.

[22] S. Weerakoon, "The "Sumudu transform" and the Laplace transform-reply," International Journal of Mathematical Education in Science and Technology, vol. 28, no. 1, pp. 159-160, 1997.

[23] F. Oberhettinger and L. Badii, Tables of Laplace Transforms, Springer, Berlin, Germany, 1973.

[24] I. Podlubny, Fractional Differential Equations, Academic Press, New York, NY, USA, 1999. 


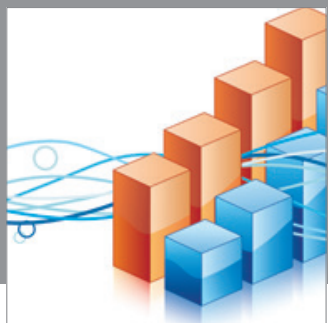

Advances in

Operations Research

mansans

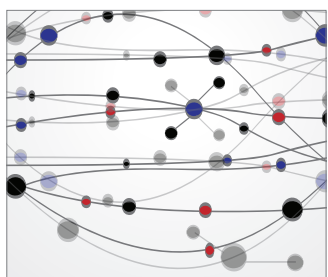

The Scientific World Journal
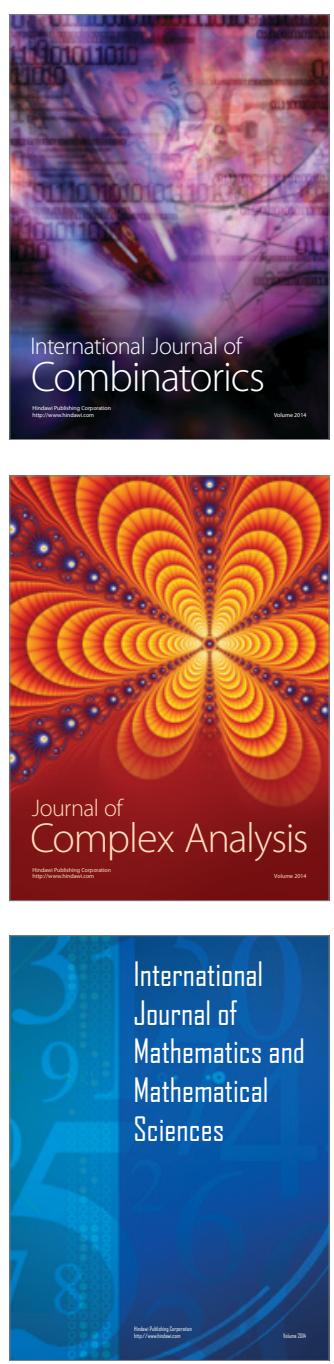
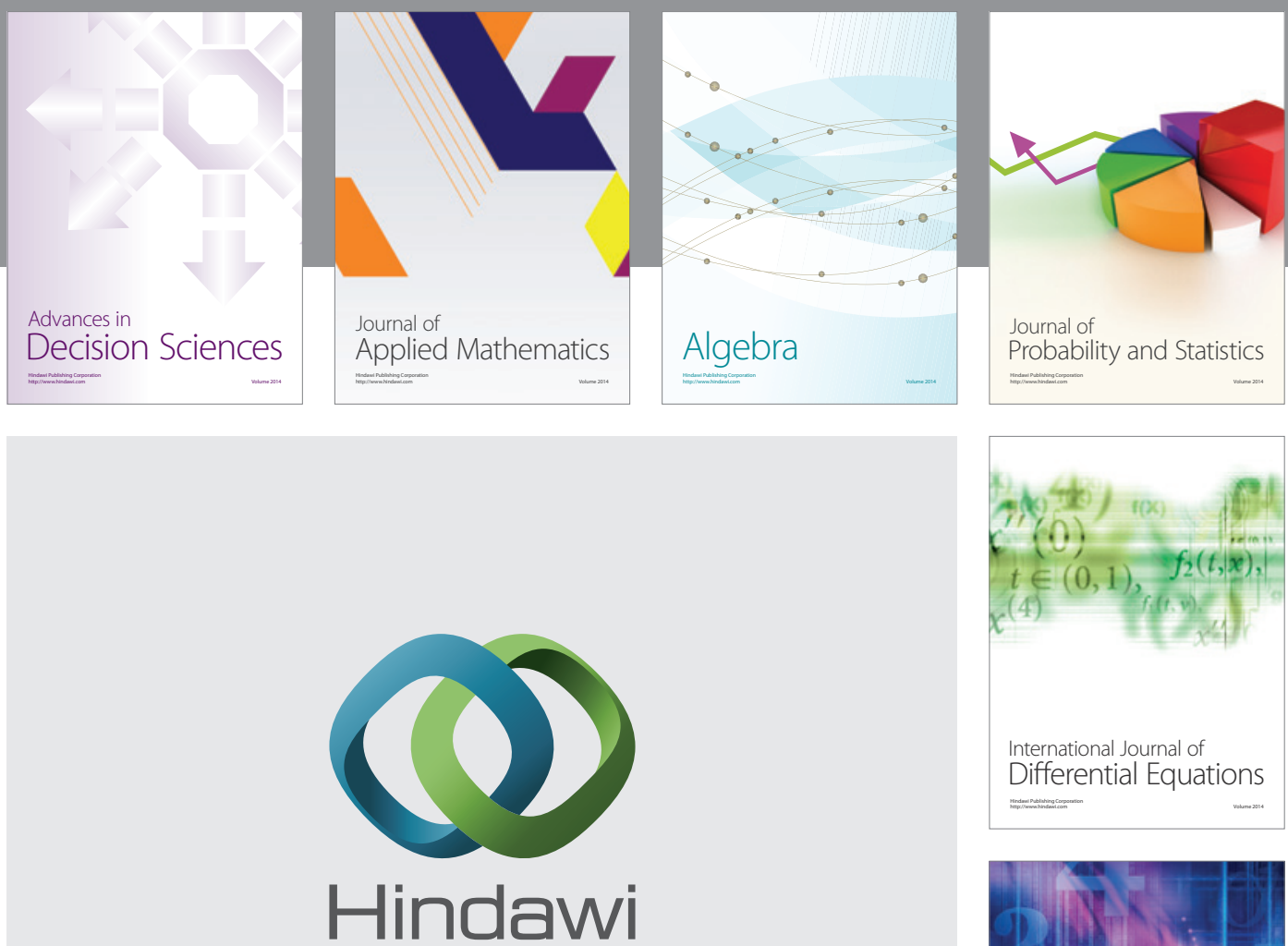

Submit your manuscripts at http://www.hindawi.com
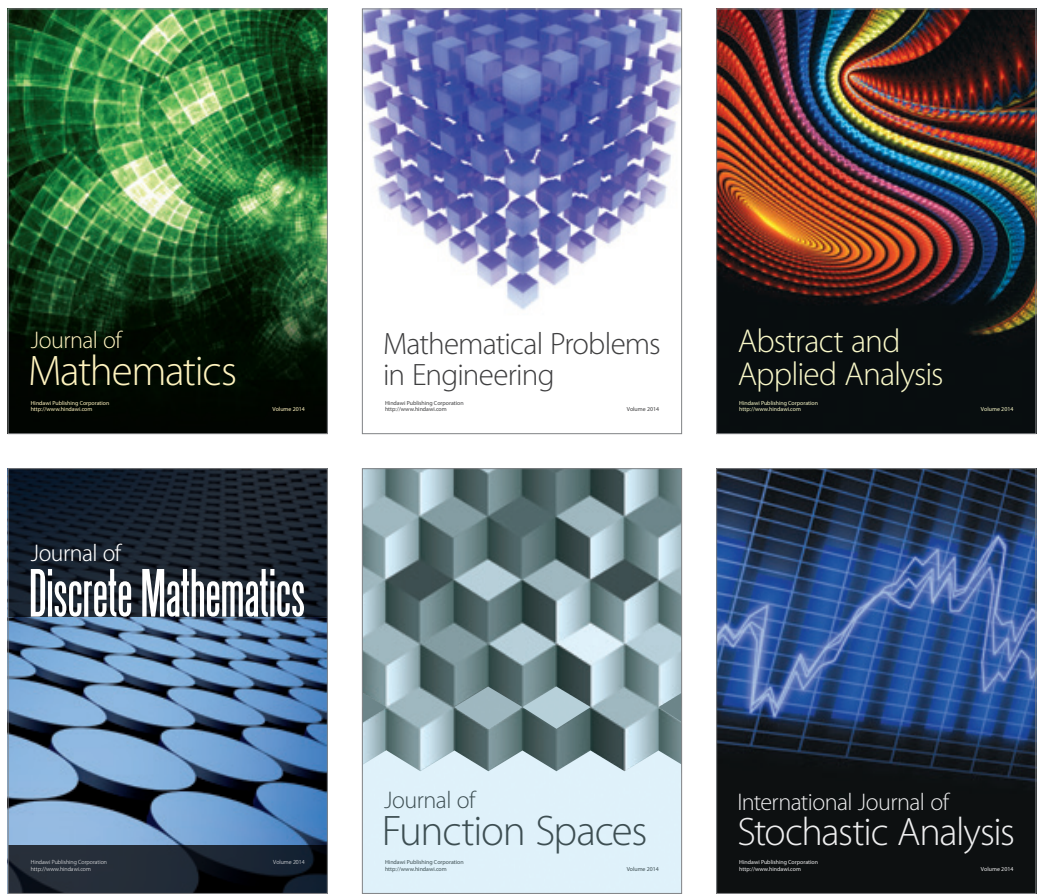

Journal of

Function Spaces

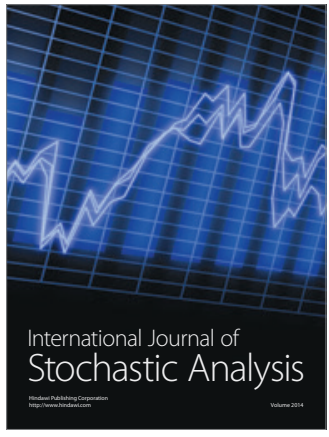

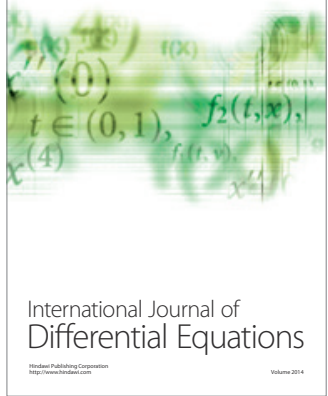
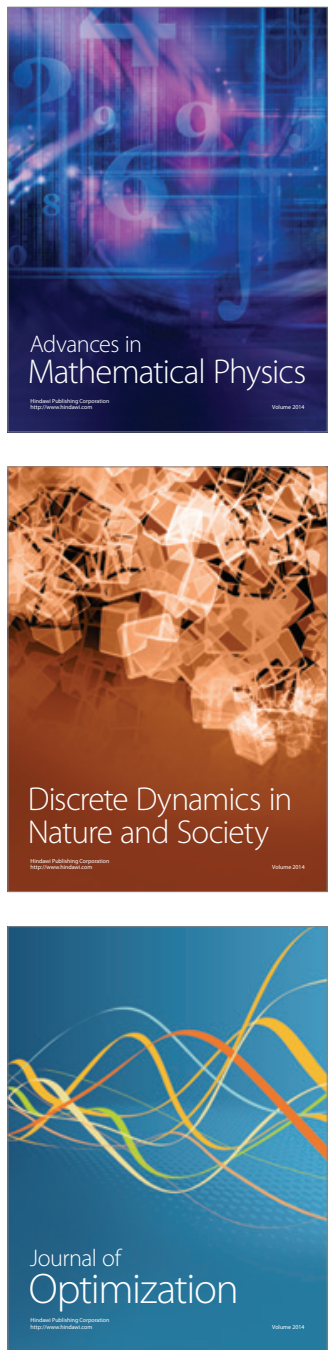